\title{
Updated meta-analysis of randomized controlled trials comparing conventional excisional haemorrhoidectomy with LigaSure for haemorrhoids
}

\author{
M.Y. Mastakov • P.G. Buettner • Y.-H. Ho
}

\begin{abstract}
Background To compare the surgical outcome of haemorrhoidectomy performed using LigaSure bipolar diathermy with conventional haemorrhoidectomy. Methods Only randomized and alternate allocated studies were included from the major electronic databases using the search terms "ligasure" and "haemorrhoids". Duration of operation, blood loss during operation, postoperative pain score, wound healing, in-hospital stay, time to return to normal activities and complications were assessed. Results The 11 trials contained a total of 1,046 patients; the largest study was based on 273 patients and two earlier studies were based on 34 patients. No significant gender mismatch between the groups was reported in any of the studies. The patients' ages were similar between groups in the studies, as was disease severity. All 11 studies reported a shorter duration of the operation when using LigaSure compared to the conventional tech-
\end{abstract}

M.Y. Mastakov · Y.-H. Ho (区)

Department of Surgery

School of Medicine

James Cook University

The Townsville Hospital, Townsville, Queensland, Australia 4814

Tel.: 61-7-47961417

Fax: +61-7-47961401

e-mail: yikhong.ho@jcu.edu.au

P.G. Buettner

School of Public Health

Tropical Medicine, and Rehabilitation Sciences

North Queensland Center for Cancer Research

James Cook University, Townsville, Australia nique $(p<0.001)$. The postoperative pain score $(p=0.001)$ and blood loss during operation $(p=0.001)$ were significantly reduced. After LigaSure haemorrhoidectomy wound healing $(p=0.004)$ and the return to normal activities $(p=0.001)$ were significantly faster than after conventional haemorrhoidectomy. However, the overall incidence of complications reported was not significantly different $(p=0.056)$. Conclusions LigaSure is an effective instrument for haemorrhoidectomy which results in less blood loss, quicker wound healing and earlier return to work.

Key words Meta-analysis · Haemorrhoids · Haemorrhoidectomy $\cdot$ LigaSure

\section{Introduction}

Definitive treatment for grade 3 and 4 haemorrhoids is by haemorrhoidectomy [1]. Excisional haemorrhoidectomy including the Milligan-Morgan technique and its modifications has been widely used [2]. However, there may be significant postoperative complications such as pain, bleeding, incontinence, and wound infections prolonged healing [3]. Recent advances in instruments such as the bipolar electrothermal device [4], circular stapler [3] and the ultrasonic scalpel [5] have provided effective alternatives, with possible reduction of postoperative pain, less blood loss, faster wound healing and a quicker return to normal activities. The LigaSure vessel sealing system (Valleylab, Boulder, CO) is a novel, haemostatic device that seals blood vessels by an optimized combination of pressure and radiofrequency ablation [6]. It ensures complete occlusion of arteries and veins up to $7 \mathrm{~mm}$ in diameter with minimal surrounding thermal spread and limit- 
ed tissue charring. Hence, the LigaSure system is potentially an effective instrument for haemorrhoid excision with minimal tissue injury resulting in reduced postoperative pain, infection rate, time for wound healing and time to return to work.

Several published randomized controlled trials have compared LigaSure with conventional excisional haemorrhoidectomy. A meta-analysis of short-term outcomes of LigaSure versus conventional haemorrhoidectomy was reported in 2007 [7]. Since then, two further randomized controlled trials have been published with substantial contributions to patient numbers $[8,9]$. We consolidated the knowledge on best practice by meta-analysis including the most recent publications. In addition, the metaanalysis was stratified to determine if effects changed when LigaSure was compared to open or to closed conventional haemorrhoidectomy techniques.

\section{Methods}

\section{Selection criteria}

Randomized controlled trials comparing LigaSure haemorrhoidectomy with excisional haemorrhoidectomy for prolapsed haemorrhoids in adults were included. Emergency situations particularly involving thrombosed internal haemorrhoids were excluded. The instrument used for excisional haemorrhoidectomy may have been scalpel, scissors, or diathermy. The haemorrhoid pedicle may have been ligated or excised by diathermy alone. Management of the wound may have been by laying open [2] or by primary closure [10]. Procedures involving stapling devices and the Harmonic Scalpel were not included in the analysis. Non-randomized studies were excluded. In addition, trials which included anal dilatation and sphincterotomy in the surgical techniques were excluded, as the addition of these procedures would potentially confound the results [11].

\section{Search strategy}

The electronic databases Medline, Embase, LigaSure manufacturer's website (http://www.ligasure.com/pages/ articallist.htm) and Cochrane Library were searched from 1996 (when LigaSure was first reported) to 2008. The search terms 'LigaSure', 'haemorrhoid', haemorrhoids', 'hemorrhoid', hemorrhoids', 'anus', 'pile', and 'piles' were used to retrieve all variants of the root term. All included studies were also entered into the PubMed 'related articles' function and the science citation index. In addition, we attempted to identify other studies by hand-searching the reference sections of these papers and by contacting known experts in the field.

\section{Study selection}

Two members of the review team (M.M., Y.-H.H.) independently assessed the titles and abstracts of all the identified studies. The individually recorded decisions of the two reviewers were compared, and any disagreements resolved by the third reviewer. Following that, two reviewers evaluated each of the eligible studies, and decided whether to include or exclude each study according to the above inclusion and exclusion criteria. Two studies (Thorbeck and Montes [12] and Chung and Wu [13]) described alternate allocation and were dealt with by conducting sensitivity analyses.

\section{Data extraction}

Two reviewers (M.M., Y.-H.H.) independently extracted details from the selected studies. The outcome measures considered were based on end-points provided in the studies. Continuous outcomes, summary estimates per group (means, medians) with measures of variability (standard deviation) or precision (standard error), confidence interval), as available, were recorded. Authors were directly contacted when case information provided in the articles was ill-defined. The third reviewer (P.G.B.) resolved discrepancies between the first two reviewers. All actual discrepancies were minor and were resolved with discussion until agreement was met. All authors agreed to assume that complications which were not explicitly mentioned in the articles did not occur. Biographical details, such as author, journal, year of publication and language, were also recorded.

\section{Quality assessment}

Two authors (P.G.B., Y.-H.H.) independently assessed the quality of the studies included in the analysis. The assessors were blinded to names, affiliations and addresses of the investigators as well as to the journals in which the trials were published. Quality assessment was based on the 17 checklist items required in the methods and results of randomised controlled trials according to the CONSORT statement [14]. The quality of the included studies was assessed on allocation concealment, blinding of participants, investigators and observers, intention-to-treat analysis, and completeness of follow-up. Agreement between the two assessors was judged using the concor- 
dance correlation coefficient $(r)$ [15]. Discordant scores were resolved based on real differences in interpretation through consensus or third-party arbitration. Resulting scores were sorted to identify any publication of lower design and reporting quality for sensitivity analysis.

\section{Statistical analysis}

For the outcome measure incidence of complications relative risk was chosen as the treatment effect measure. For duration of operation, in-hospital stay, postoperative pain score, blood loss during operation, wound healing, and time to return to normal activities, standardized differences in means were used as treatment effect measures. When necessary, standard deviations were estimated from range values provided. Wang et al. reported standard errors as their measures of dispersion (confirmed after personal communication) [16]. Quantitative metaanalyses were conducted using calculated standard deviations for this study based on the reported standard error. Combined overall effect measures were calculated for random effect model assumptions and are presented with 95\% confidence intervals (95\% CI). Statistical heterogeneity, the variation in outcomes between studies, was assessed using the chi-squared distributed Q-statistic. Sensitivity analyses were carried out by excluding the two studies $[12,13]$ which used alternate allocation, and hence considered to be of lower quality.

Quantitative meta-analyses were calculated stratified by type of conventional technique (open or closed haemorrhoidectomy). To judge publication bias, the classic fail-safe $N$ was calculated for duration of operation, postoperative pain score, blood loss during operation, time to return to normal activities, and incidence of complications only, as the calculation requires a minimum of three studies. Throughout analysis a significance level of 0.05 was assumed. Statistical analysis was conducted using the software program Comprehensive Meta Analysis, Version 2 (Biostat, Englewood, NJ).

\section{Results}

Identification and characteristics of studies

Figure 1 summarizes the process of identifying eligible clinical trials. Of 30 citations identified from electronic databases and by hand searching, 13 were selected for full text assessment. However, two studies were found to be the long-term follow-up of previous randomized controlled trials $[17,18]$. Finally, 11 trials published in peerreviewed journals between 2002 and 2007 were included $[8,9,12,13,16,19-24]$. The largest study was based on 273 patients [9] and two studies were based on 34 patients (Table 1) [21, 23]. In total 579 (of 1,046) recruited patients came from Italian centres $[8,9,20]$. All but one study [9] were single-centre studies. Agreement amongst authors for study inclusion was $100 \%$.

\section{Quality of studies}

The studies of Thorbeck and Montes [12] and Chung and $\mathrm{Wu}$ [13] were identified to be of lesser design quality, as both studies used alternate allocation instead of randomization and both studies had no blinded outcome assessment. Overall, only five studies reported some form of blinding, but only Muzi et al. [8] and Chung et al. [22] reported that all outcome assessment was blinded. Agreement on the quality of the nine studies between both assessors was reasonably high $(r=0.76 ; 95 \%$ CI $[0.42,0.91])$.

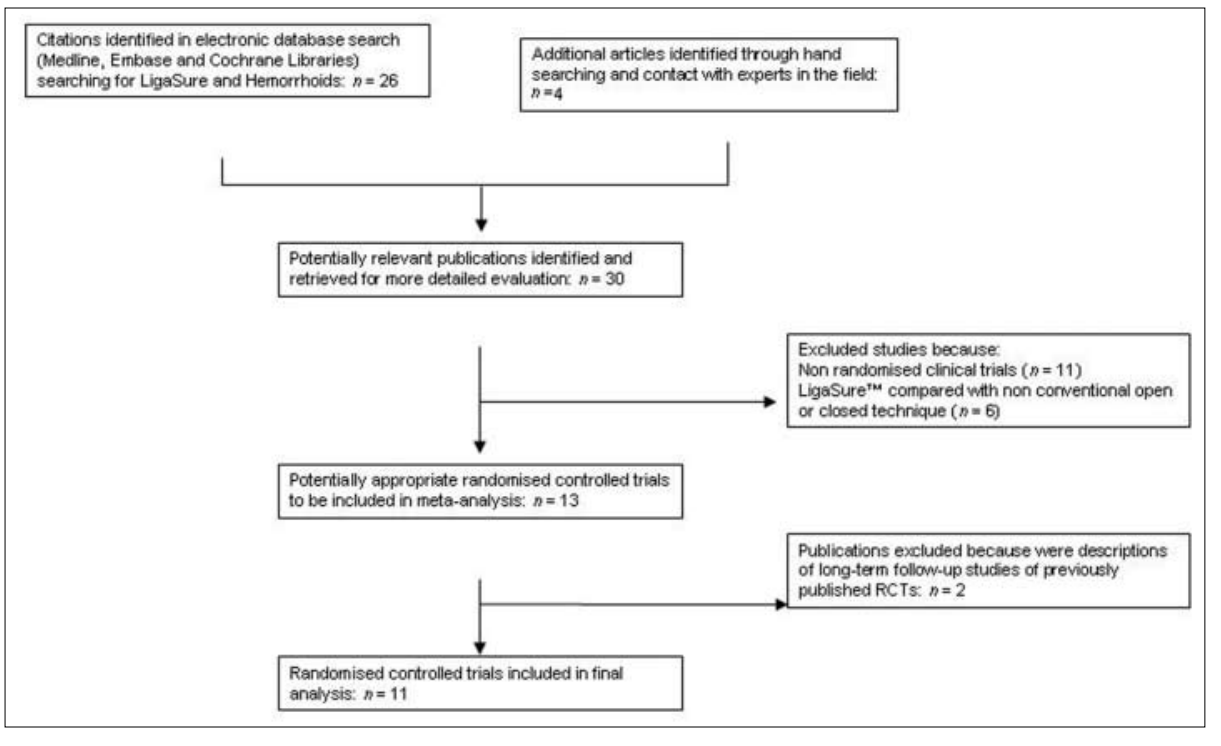

Fig. 1 Flow diagram for study selection 
Table 1 Eleven randomised controlled trials comparing LigaSure $(L S)$ with conventional haemorrhoidectomy $(C H)$

\begin{tabular}{|c|c|c|c|c|c|c|c|c|}
\hline Reference & Country & Year & Study details & $\begin{array}{l}\text { Conventional } \\
\text { technique }\end{array}$ & $\begin{array}{l}\text { Number } \\
\text { of patients }\end{array}$ & Age (years) & $\begin{array}{l}\text { Number } \\
\text { of male } \\
\text { patients }\end{array}$ & $\begin{array}{l}\text { Number } \\
\text { of patients } \\
\text { with grade } \\
\text { III (IV) } \\
\text { hemorrhoids }\end{array}$ \\
\hline 19 & UK & 2002 & $\begin{array}{l}\text { Truly random, } \\
\text { follow-up } \\
\text { assessment } \\
\text { blinded }\end{array}$ & $\begin{array}{l}\text { Open diathermy, } \\
\text { pedicles transfixed }\end{array}$ & LS 20, CH 20 & $\begin{array}{l}\text { Mean (range): } \\
\text { LS } 48 \text { (26-75), } \\
\text { CH } 43 \text { (33-77) }\end{array}$ & $\begin{array}{l}\text { LS 11, } \\
\text { CH } 10\end{array}$ & $\begin{array}{l}\text { LS } 16(4), \\
\text { CH } 16(4)\end{array}$ \\
\hline 20 & Italy & 2002 & $\begin{array}{l}\text { Truly random, } \\
\text { not blinded }\end{array}$ & $\begin{array}{l}\text { Open diathermy, } \\
\text { pedicles not ligated }\end{array}$ & LS 29, CH 27 & $\begin{array}{l}\text { Mean }(\mathrm{SD})^{\mathrm{a}} \text { : } \\
\text { LS } 52(9.9) \text {, } \\
\text { CH } 48(10.8)\end{array}$ & $\begin{array}{l}\mathrm{LS} 13, \\
\mathrm{CH} 17^{\mathrm{a}}\end{array}$ & $\begin{array}{l}\text { LS } 14(15), \\
\text { CH } 13(14)^{\mathrm{a}}\end{array}$ \\
\hline 21 & UK & 2002 & $\begin{array}{l}\text { Truly random, } \\
\text { patients blinded }\end{array}$ & $\begin{array}{l}\text { Open diathermy, } \\
\text { pedicles not ligated }\end{array}$ & LS 18, CH 16 & $\begin{array}{l}\text { Mean (range): } \\
\text { LS } 44 \text { (33-58), } \\
\text { CH } 49 \text { (30-73) }\end{array}$ & LS 6, CH 6 & NR \\
\hline 2 & Spain & 2002 & $\begin{array}{l}\text { Alternate } \\
\text { allocation, } \\
\text { not blinded }\end{array}$ & $\begin{array}{l}\text { Open diathermy, } \\
\text { pedicles not ligated }\end{array}$ & LS 56, CH 56 & Overall mean: 50 & Overall 66 & NR \\
\hline 22 & Hong Kong & 2002 & $\begin{array}{l}\text { Truly random, } \\
\text { double blinded }\end{array}$ & $\begin{array}{l}\text { Open, scissors } \\
\text { excision, } \\
\text { pedicles transfixed }\end{array}$ & LS 30, CH 27 & $\begin{array}{l}\text { Mean }(\mathrm{SD})^{\mathrm{a}} \text { : } \\
\text { LS } 50.7(12.2) \text {, } \\
\text { CH } 44.7(14.5)\end{array}$ & $\begin{array}{l}\mathrm{LS} \mathrm{16}, \\
\mathrm{CH} 13^{\mathrm{a}}\end{array}$ & $\begin{array}{l}\text { LS 30, } \\
\text { CH } 27\end{array}$ \\
\hline 13 & Taiwan & 2003 & $\begin{array}{l}\text { Alternate } \\
\text { allocation, } \\
\text { not blinded }\end{array}$ & $\begin{array}{l}\text { Closed Ferguson, } \\
\text { scissor excision }\end{array}$ & LS 30, CH 31 & $\begin{array}{l}\text { Mean (SD)a: } \\
\text { LS } 47(16.6), \\
\text { CH } 44(10.4)\end{array}$ & $\begin{array}{l}\mathrm{LS} \mathrm{18,} \\
\mathrm{CH} \mathrm{12}\end{array}$ & $\begin{array}{l}\mathrm{LS} 8(22), \\
\mathrm{CH} 8(23)^{\mathrm{a}}\end{array}$ \\
\hline 23 & UK & 2003 & $\begin{array}{l}\text { Truly random, } \\
\text { not blinded }\end{array}$ & $\begin{array}{l}\text { Closed modified } \\
\text { Ferguson } \\
\text { (monopolar) } \\
\text { diathermy }\end{array}$ & LS 17, CH 17 & $\mathrm{NR}^{\mathrm{a}}$ & MF ratio $1: 1$ & $\mathrm{NR}^{\mathrm{a}}$ \\
\hline 16 & Taiwan & 2006 & $\begin{array}{l}\text { Truly random, } \\
\text { pain assessment } \\
\text { blinded }\end{array}$ & $\begin{array}{l}\text { Closed Ferguson } \\
\text { diathermy }\end{array}$ & $\mathrm{LS} 42, \mathrm{CH} 42$ & $\begin{array}{l}\text { Mean (SEM) })^{\mathrm{a}} \text { : } \\
\text { LS } 47(2.4) \text {, } \\
\text { CH } 47(2.3)\end{array}$ & 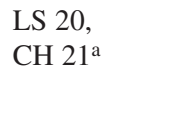 & $\begin{array}{l}\text { LS } 34(8), \\
\text { CH } 35(7)^{\mathrm{a}}\end{array}$ \\
\hline 24 & Thailand & 2006 & $\begin{array}{l}\text { Truly random, } \\
\text { not blinded }\end{array}$ & $\begin{array}{l}\text { Fansler } \\
\text { (semiclosed/open), } \\
\text { scissors excision }\end{array}$ & LS 23, CH 22 & $\begin{array}{l}\text { Mean (SD)a: } \\
\text { LS } 41.9(12.9) \text {, } \\
\text { CH } 45.7(14.6)\end{array}$ & $\begin{array}{l}\mathrm{LS} \mathrm{12}, \\
\mathrm{CH} 12^{\mathrm{a}}\end{array}$ & $\begin{array}{l}\operatorname{LS} 20(3), \\
\mathrm{CH} 21(1)^{\mathrm{a}}\end{array}$ \\
\hline 8 & Italy & 2007 & $\begin{array}{l}\text { Truly random, } \\
\text { outcome } \\
\text { assessment } \\
\text { blinded }\end{array}$ & $\begin{array}{l}\text { Open diathermy, } \\
\text { scissors excision, } \\
\text { pedicles } \\
\text { not transfixed }\end{array}$ & LS 125, CH 125 & $\begin{array}{l}\text { Mean (range) })^{a} \text { : } \\
\text { LS } 47 \text { (21-66), } \\
\text { CH } 47(25-68)\end{array}$ & $\begin{array}{l}\mathrm{LS} 60 \\
\mathrm{CH} \mathrm{53}\end{array}$ & $\begin{array}{l}\text { LS } 88(37), \\
\text { CH } 81(44)^{\mathrm{a}}\end{array}$ \\
\hline 9 & Italy & 2007 & $\begin{array}{l}\text { Truly random, } \\
\text { not blinded }\end{array}$ & $\begin{array}{l}\text { Open diathermy, } \\
\text { pedicles not ligated }\end{array}$ & LS 146, CH 127 & $\begin{array}{l}\text { Median }{ }^{\mathrm{a}} \text { : LS } 49 \text {, } \\
\text { CH48 }\end{array}$ & $\begin{array}{l}\mathrm{LS} 80, \\
\mathrm{CH} \mathrm{76}{ }^{\mathrm{a}}\end{array}$ & $\begin{array}{l}\text { LS } 44(59) \text {, } \\
\text { CH } 50(60)^{\mathrm{a}}\end{array}$ \\
\hline
\end{tabular}

$N R$, not reported

aNo significant difference between the two groups

Patient characteristics of eligible studies

A total of 1,046 patients were reported in the 11 eligible studies (Table 1). The percentage of males varied from $33 \%$ [21] to $60 \%$ [13] in the LigaSure group, and from $38 \%$ [21] to $63 \%$ [20] in the conventional group. No significant gender mismatch between the groups was reported in any of the studies. The patient ages were similar between groups in the studies, as was disease severity. No significant discrepancies in patient characteristics between groups were reported in any of the studies. None of the 11 studies included in the analysis had recorded the duration of symptoms. In six of the seven studies in which the number of haemorrhoids excised per patient was reported, an average of three haemorrhoids were excised in either group (Table 2); Pattana-Arun et al. excised a mean of 2.9 haemorrhoids in the LigaSure group and 2.2 in the conventional group ( $p=0.039)$ [24]. Four studies did not record the number of haemorrhoids excised [8, 12, 20, 23].

\section{Haemorrhoidectomy procedures}

The operations were performed under general, spinal or epidural anaesthesia. In all the 11 studies reported, the LigaSure haemorrhoidectomy was performed using the same incision and along the same tissue plane, with similar amounts of haemorrhoidal tissue excised as conven- 
Table 2 Operative and postoperative parameters of 11 randomized controlled trials comparing LigaSure $(L S)$ with conventional haemorrhoidectomy $(\mathrm{CH})$

\begin{tabular}{|c|c|c|c|c|c|}
\hline Reference & $\begin{array}{l}\text { Number } \\
\text { of haemorrhoids } \\
\text { excised }\end{array}$ & $\begin{array}{l}\text { Duration of } \\
\text { operation (min) }\end{array}$ & $\begin{array}{l}\text { Blood loss } \\
\text { during operation (ml) }\end{array}$ & $\begin{array}{l}\text { First bowel } \\
\text { movement (days) }\end{array}$ & In-hospital stay (days) \\
\hline 19 & $\begin{array}{l}\text { Median: LS 3, } \\
\text { CH 3 } 3^{\text {a }}\end{array}$ & $\begin{array}{l}\text { Median (range): } \\
\text { LS } 10(8-11) \text {, } \\
\text { CH } 20(18-25)^{*}\end{array}$ & $\begin{array}{l}\text { Median (range): } \\
\text { LS } 0(0-5) \text {, } \\
\text { CH } 20(12-22)^{*}\end{array}$ & $\begin{array}{l}\text { Median (range): } \\
\text { LS } 2(1-5) \text {, } \\
\text { CH } 2(1-4)^{\mathrm{a}}\end{array}$ & $\begin{array}{l}\text { Median (range): } \\
\text { LS } 0(0-1) \text {, CH } 0(0-2)^{*} \\
\text { LS } 0(0-5), \text { CH } 20(12-22)^{*}\end{array}$ \\
\hline 20 & NR & $\begin{array}{l}\text { Mean (SD): } \\
\text { LS 9.2 (3.4), } \\
\text { CH } 12.1(3.6)^{*}\end{array}$ & NR & Mean: LS 2, CH $2^{\mathrm{a}}$ & $\begin{array}{l}\text { Mean (range): } \\
\text { LS } 1.8(1,3), \text { CH } 1.3(1-4)^{a}\end{array}$ \\
\hline 21 & $\begin{array}{l}\text { Median: LS 3, } \\
\text { CH } 3\end{array}$ & $\begin{array}{l}\text { Median (range): } \\
\text { LS 5.1 (2-9), } \\
\text { CH 9.2 (7.6-14.1)* }\end{array}$ & NR & NR & $\begin{array}{l}\text { Median (range): } \\
\text { LS } 1(0-1), \text { CH } 1(1-1)\end{array}$ \\
\hline 12 & NR & $\begin{array}{l}\text { Mean }(\mathrm{SD}) \\
\text { per cushion: } \\
\text { LS } 1.7(0.3) \\
\text { CH } 5.2(1.1)^{*}\end{array}$ & $\begin{array}{l}\text { Mean: LS 0, } \\
\text { CH } 10\end{array}$ & NR & Median: LS 2, CH 2 \\
\hline 22 & All patients: 3 & $\begin{array}{l}\text { Mean (SD): } \\
\text { LS 15.2 (7.1), } \\
\text { CH 17.6 (8.3) }\end{array}$ & $\begin{array}{l}\text { Mean (SD): } \\
\text { LS } 13.5(27.5) \text {, } \\
\text { CH } 46.5(27.5)^{*}\end{array}$ & $\begin{array}{l}\text { Mean }(\mathrm{SD}) \text { : } \\
\text { LS 2.2 (1.1), } \\
\text { CH 2.5 (2.4) }\end{array}$ & $\begin{array}{l}\text { Mean (SD): } \\
\text { LS } 3.6(2), \text { CH } 3.8(2)^{a}\end{array}$ \\
\hline 13 & $\begin{array}{l}\text { Median (range): } \\
\text { LS } 3(3-4) \text {, } \\
\text { CH } 3(3-4)^{\text {a }}\end{array}$ & $\begin{array}{l}\text { Mean (SD): } \\
\text { LS 15.0 (5.4), } \\
\text { CH 21.2 (8.2)* }\end{array}$ & $\begin{array}{l}\mathrm{NR} \\
\text { ("LS less bleeding") }\end{array}$ & NR & $\begin{array}{l}\text { Mean (SD): } \\
\text { LS } 3.2(0.8), \text { CH } 3.5(1.0)^{\mathrm{a}}\end{array}$ \\
\hline 23 & NR & $\begin{array}{l}\text { Median (range): } \\
\text { LS } 6 \text { (4-10), } \\
\text { CH } 11(7-20)^{*}\end{array}$ & NR & NR & $\begin{array}{l}\text { Mean (range): } \\
\text { LS } 1(1-5), \text { CH } 1(1-5)\end{array}$ \\
\hline 16 & $\begin{array}{l}\text { Median (range): } \\
\text { LS } 3(2-4) \text {, } \\
\text { CH } 3(2-4)^{\mathrm{a}}\end{array}$ & $\begin{array}{l}\text { Mean (SEM): } \\
\text { LS } 11.3(0.4) \text {, } \\
\text { CH } 34.2(0.7)^{*}\end{array}$ & $\begin{array}{l}\text { Mean (SEM): } \\
\text { LS 1.8 (0.3), } \\
\text { CH 25.7 (1.3)* }\end{array}$ & NR & $\begin{array}{l}\text { Mean (SEM): } \\
\text { LS } 2.2(0.1), \text { CH } 2.9(0.1)^{*}\end{array}$ \\
\hline 24 & $\begin{array}{l}\text { Mean (SD): } \\
\text { LS } 2.9(1.4) \\
\text { CH } 2.2(0.9)^{*}\end{array}$ & $\begin{array}{l}\text { Mean (SD): } \\
\text { LS } 21.7(11.8) \text {, } \\
\text { CH } 35.7(14.3)^{*}\end{array}$ & $\mathrm{NR}$ & NR & NR \\
\hline 8 & NR & $\begin{array}{l}\text { Mean (range): } \\
\text { LS } 11.5(8-33) \text {, } \\
\text { CH } 20(19.5-48)^{*}\end{array}$ & NR & NR & $\begin{array}{l}\text { Mean (range): } \\
\text { LS 0.3 }(0.25-0.5) \text {, } \\
\text { CH } 0.4(0.25-0.5)^{\mathrm{a}}\end{array}$ \\
\hline 9 & $\begin{array}{l}\text { Mean: } \\
\text { LS 3.2, CH } 3.1\end{array}$ & Mean: LS 30, CH 31a & NR & $\begin{array}{l}\text { Mean: } \\
\text { LS 1.5, CH } 1.7^{\mathrm{a}}\end{array}$ & $\begin{array}{l}\text { Mean: } \\
\text { LS } 21 \text { hours, CH } 1 \text { daya }\end{array}$ \\
\hline
\end{tabular}

$N R$, not reported

* Significant difference between the two groups

${ }^{\text {aNo }}$ significant difference between the two groups

tional excisional haemorrhoidectomy except that the LigaSure bipolar cautery device was used instead. The technique has been well described in all reported trials $[8,9,12,13,16,19-24]$. The wounds were laid open after conventional excisional haemorrhoidectomy in seven studies (see Table 1) [8, 9, 12, 19-22]. A diathermy technique was used and the pedicles were not suturetransfixed except in the patients of Jayne et al. and Chung et al. [19, 22]. The wounds were closed after conventional excisional haemorrhoidectomy in three studies $[13,16$, 23]. Diathermy was used in the excision except in three studies, in which scissors were used for excision [13, 22, 24]. In the study of Pattana-Arun et al. the anorectal mucosal wounds were closed with sutures, but the perianal skin wounds were left laid open after conventional excisional haemorrhoidectomy [24].
Operative and postoperative parameters

The operative and postoperative parameters in the 11 randomized controlled trials are shown in Table 2. Quantitative meta-analysis confirmed that LigaSure haemorrhoidectomy took a significantly shorter time than conventional haemorrhoidectomy $(p<0.001$; Table 3 and Fig. 2). It would take 1,049 studies to negate this significant result (classic fail-safe $N$ ). Blood loss during operation was reported quantitatively by Jayne et al. [19], Thorbeck and Montes [12], Chung et al [22] and Wang et al. [16]; while Chung and Wu [13] noted that less bleeding occurred during LigaSure haemorrhoidectomy without further quantification (Table 2). Meta-analysis showed that blood loss during LigaSure haemorrhoidectomy was significantly 
Table 3 Postoperative pain, postoperative analgesic use, wound healing, return to normal activities and follow-up time reported in randomized controlled trials comparing LigaSure $(L S)$ with conventional haemorrhoidectomy $(\mathrm{CH})$

\begin{tabular}{|c|c|c|c|c|c|}
\hline Reference & $\begin{array}{l}\text { Postoperative pain } \\
\text { score }^{\mathrm{d}}\end{array}$ & $\begin{array}{l}\text { Postoperative } \\
\text { analgesic use }\end{array}$ & Wound healing (days) & $\begin{array}{l}\text { Return to normal } \\
\text { activities }\end{array}$ & $\begin{array}{l}\text { Median follow-up } \\
\text { (months) }\end{array}$ \\
\hline 19 & $\begin{array}{l}\text { Median (range), } \\
\text { day 1: LS } 5 \text { (1-9), } \\
\text { CH } 7(4-9)^{\mathrm{a}}\end{array}$ & $\mathrm{NR}^{\mathrm{a}}$ & NR & $\begin{array}{l}\text { Back at work } \\
\text { at } 3 \text { weeks }{ }^{\text {a: }} \\
\text { LS } 17 \text { of } 20 \text {, } \\
\text { CH } 15 \text { of } 20\end{array}$ & $\begin{array}{l}\text { LS 3, CH 3; } \\
\text { long-term }{ }^{\text {b: }} \\
\text { LS 37, CH } 36\end{array}$ \\
\hline 20 & $\begin{array}{l}\text { Median (range), } \\
\text { day 1: LS } 4.7(2-8) \text {, } \\
\text { CH 5.2 }(2-8)^{\mathrm{a}}\end{array}$ & $\begin{array}{l}\text { Median (range) } \\
\text { number used: } \\
\text { LS } 14.1 \text { (10-20), } \\
\text { CH } 16.8(12-22)^{*}\end{array}$ & $\begin{array}{l}\text { Median (range): } \\
\text { LS } 16.3 \text { (14-22), } \\
\text { CH } 37.5 \text { (25-52)* }\end{array}$ & $\begin{array}{l}\text { Median days (range): } \\
\text { LS } 8.3(5-14) \text {, } \\
\text { CH } 18.3(10-30)^{*}\end{array}$ & LS 6, CH 6 \\
\hline 21 & $\begin{array}{l}\text { Median (range): } \\
\text { LS 5.2 (2-8), } \\
\text { CH 4.6 (1-8.4) }\end{array}$ & $\begin{array}{l}\text { Median (range) } \\
\text { 7-days tramadol (mg): } \\
\text { LS } 850(0-2,750) \text {, } \\
\text { CH } 1600(0-2100)^{*}\end{array}$ & NR & NR & $\begin{array}{l}\text { LS } 1.5, \text { CH } 1.5 \text {; } \\
\text { long-termc: } \\
\text { LS } 15, \text { CH } 16\end{array}$ \\
\hline 12 & $\begin{array}{l}\text { Mean (SD), } \\
\text { day 1: LS } 2.3(0.8) \text {, } \\
\text { CH } 6.9(0.8)^{*}\end{array}$ & $\begin{array}{l}\text { Mean (SD) } \\
\text { no. of dipyrone tablets: } \\
\text { LS } 2.1(0.5) \text {, } \\
\text { CH } 4.6(0.8)^{*}\end{array}$ & LS "better and faster" & NR & LS 6, CH 6 \\
\hline 22 & $\begin{array}{l}\text { Mean (SD): } \\
\text { LS } 4.5(2) \text {, } \\
\text { CH } 4.0(2.3)^{\mathrm{a}}\end{array}$ & $\begin{array}{l}\text { Mean (SD) pethidine } \\
\text { injections }{ }^{\mathrm{a}} \text { and } \\
\text { dologesic tablets }^{\mathrm{a}} \text { : } \\
\text { LS } 0.7(1) \text { and } \\
12.6(10.7) \text {, } \\
\text { CH } 1.2(2.1) \text { and } \\
13.3(11.1)\end{array}$ & $\mathrm{CH}$ impaired at 4 weeks & $\begin{array}{l}\text { Mean (SD) days: } \\
\text { LS } 11.6(5.4) \text {, } \\
\text { CH } 10.3(4.5)^{\mathrm{a}}\end{array}$ & LS $3, \mathrm{CH} 3$ \\
\hline 13 & $\begin{array}{l}\text { Mean (SD), } \\
\text { day 1: LS } 6.5(0.4) \text {, } \\
\text { CH } 8(0.5)^{*}\end{array}$ & NR & $\begin{array}{l}\text { Both groups } \\
\text { "complete after } 6 \text { weeks" }\end{array}$ & $\begin{array}{l}\text { Median: } \\
\text { LS 1-2 weeks, } \\
\text { CH 1-2 weeks }\end{array}$ & $\mathrm{LS} 4, \mathrm{CH} 4$ \\
\hline 23 & $\begin{array}{l}\text { Median (range), } \\
\text { day 1: LS 2.5 (0-6), } \\
\text { CH 7.5 (6-9)* }\end{array}$ & NR & NR & NR & LS 3, CH 3 \\
\hline 16 & $\begin{array}{l}\text { Mean (SEM): } \\
\text { LS 5.1 }(0.2) \\
\text { CH } 7.2(0.2)^{*}\end{array}$ & $\begin{array}{l}\text { Parenteral: } \\
\text { LS 12, CH 32* }\end{array}$ & $\begin{array}{l}\text { Both groups } \\
\text { "complete after } 6 \text { weeks"a }\end{array}$ & $\begin{array}{l}\text { Mean days (SEM): } \\
\text { LS } 8.8(0.2) \text {, } \\
\text { CH } 13.7(0.4)^{*}\end{array}$ & $\mathrm{LS} 2, \mathrm{CH} 2$ \\
\hline 24 & $\begin{array}{l}\text { Median, day 1: } \\
\text { LS 3.65, CH 3.14 }\end{array}$ & $\begin{array}{l}\text { Mean total dose }(\mathrm{mg}) \\
\text { of pethidine: LS } 65 \text {, } \\
\mathrm{CH} 75^{\mathrm{a}}\end{array}$ & $\begin{array}{l}\text { Both groups } \\
\text { "complete after } 4 \text { weeks"a }\end{array}$ & $\mathrm{NR}$ & LS 1, CH 1 \\
\hline 8 & $\begin{array}{l}\text { Mean (range), } \\
\text { day 1: LS 1.5 (0-3), } \\
\text { CH 3.3 (2-6)* }\end{array}$ & NR & $\begin{array}{l}\text { Mean (range): } \\
\text { LS } 14.8(10-21) \text {, } \\
\text { CH } 25.6(14-40)^{*}\end{array}$ & $\begin{array}{l}\text { Mean (range): } \\
\text { LS } 12.3(5-21) \\
\text { CH } 16.4(10-30)^{*}\end{array}$ & LS 36, CH 36 \\
\hline 9 & $\begin{array}{l}\text { Median, day 1: } \\
\text { LS 2.8, CH } 3.4\end{array}$ & $\begin{array}{l}\text { Median number } \\
\text { of tablets, day } 3 \text { : } \\
\text { LS } 0.5, \text { CH } 1^{*}\end{array}$ & $\begin{array}{l}\text { Incomplete healing } \\
\text { after } 4 \text { weeks: } \\
\text { LS } 9 \text { patients, } \\
\text { CH } 8 \text { patients }\end{array}$ & $\begin{array}{l}\text { Median days: } \\
\text { LS 10.8, CH 14.5* }\end{array}$ & LS 1, CH 1 \\
\hline
\end{tabular}

$N R$, not reported

*Significant difference between the two groups

aNo significant difference between the two groups

${ }^{b}$ Median follow-up time, Peters et al. [16]

${ }^{c}$ Median follow-up time, Lawes et al. [17]

dPostoperative pain score: 0 no pain, 10 most severe pain

less than during conventional haemorrhoidectomy ( $p=0.001$; Table 3$)$ and it would take 355 studies to negate this result. The time to first postoperative bowel movement was recorded in four studies, none of which reported any significant differences between LigaSure and conventional haemorrhoidectomy (Table 2). Ten of the 11 studies reported length of stay in hospital.
Quantitative meta-analysis showed no significant difference between LigaSure and conventional haemorrhoidectomy with respect to hospitalization $(p=0.205$; Table 3).

Postoperative pain and postoperative analgesic use after LigaSure and conventional haemorrhoidectomy are shown in Table 4. Eight studies reported pain 


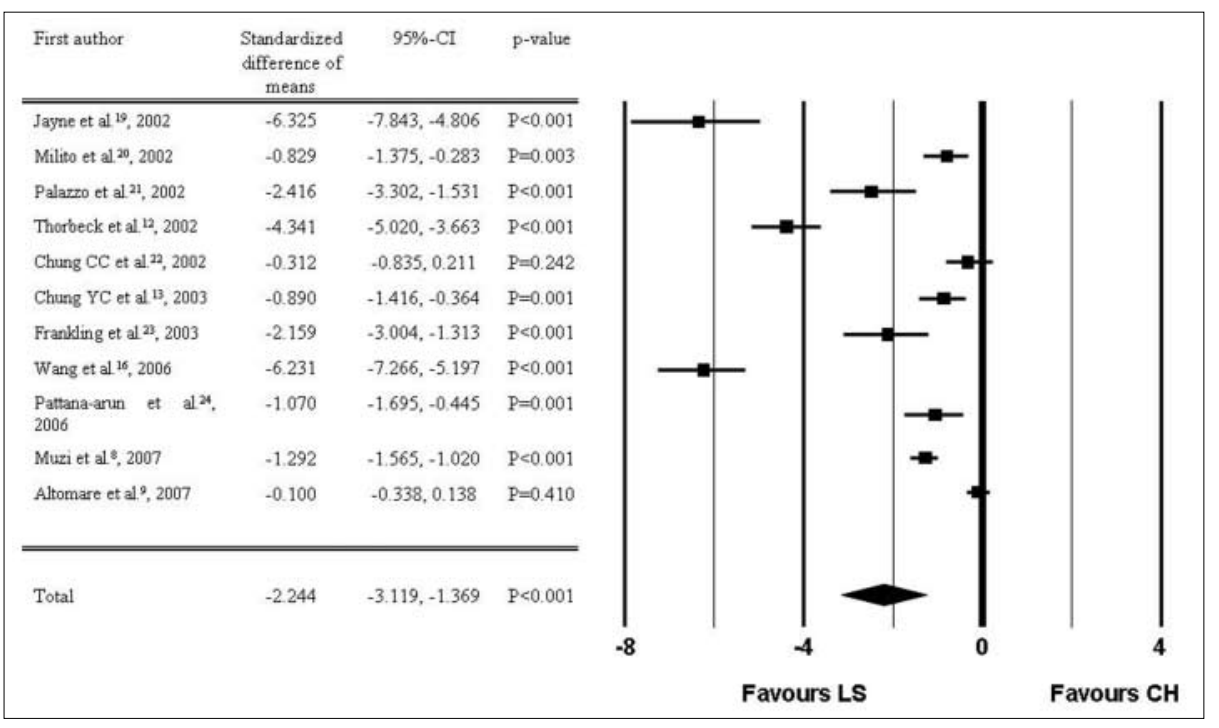

Fig. 2 Forest plot of duration of operation ( $L S$, LigaSure haemorrhoidectomy; $\mathrm{CH}$, conventional haemorrhoidectomy)

Table 4 Postoperative complications reported by 11 randomized controlled trials comparing LigaSure $(L S)$ with conventional haemorrhoidectomy $(\mathrm{CH})$

\begin{tabular}{|c|c|c|c|c|c|c|c|c|c|c|}
\hline Reference & $\begin{array}{l}\text { Number } \\
\text { of patients }\end{array}$ & Bleeding & $\begin{array}{l}\text { Urinary } \\
\text { retention }\end{array}$ & $\begin{array}{l}\text { Faecal } \\
\text { impaction }\end{array}$ & Fissure & $\begin{array}{l}\text { Nausea/ } \\
\text { vomit }\end{array}$ & $\begin{array}{l}\text { Anal } \\
\text { stenosis }\end{array}$ & $\begin{array}{l}\text { Poor wound } \\
\text { healing }^{\text {a }}\end{array}$ & Incontinence & $\begin{array}{l}\text { All } \\
\text { complications }\end{array}$ \\
\hline 19 & $\begin{array}{l}\text { LS 20, } \\
\text { CH } 20\end{array}$ & $\begin{array}{l}\text { LS } 1, \\
\text { CH } 1\end{array}$ & $\begin{array}{l}\text { LS } 1, \\
\text { CH } 0\end{array}$ & $\begin{array}{l}\text { LS } 0, \\
\text { CH } 0\end{array}$ & $\begin{array}{l}\text { LS 1, } \\
\text { CH } 1\end{array}$ & $\begin{array}{l}\text { LS } 0, \\
\text { CH } 0\end{array}$ & $\begin{array}{l}\text { LS } 0, \\
\text { CH } 0\end{array}$ & LS $0, \mathrm{CH} 0$ & LS $0, \mathrm{CH} 0$ & $\mathrm{LS} 3, \mathrm{CH} 2$ \\
\hline 20 & $\begin{array}{l}\text { LS } 29, \\
\text { CH } 27\end{array}$ & $\begin{array}{l}\text { LS 1, } \\
\mathrm{CH} 2\end{array}$ & $\begin{array}{l}\text { LS 1, } \\
\text { CH } 1\end{array}$ & $\begin{array}{l}\text { LS 2, } \\
\text { CH } 1\end{array}$ & $\begin{array}{l}\text { LS } 0 \text {, } \\
\text { CH } 0\end{array}$ & $\begin{array}{l}\text { LS } 0 \text {, } \\
\text { CH } 0\end{array}$ & $\begin{array}{l}\text { LS } 0, \\
\text { CH } 0\end{array}$ & LS $0, \mathrm{CH} 0$ & LS $0, \mathrm{CH} 0$ & $\mathrm{LS} 4, \mathrm{CH} 4$ \\
\hline 21 & $\begin{array}{l}\text { LS } 18, \\
\text { CH } 16\end{array}$ & $\begin{array}{l}\text { LS } 0 \\
\text { CH2 }\end{array}$ & $\begin{array}{l}\text { LS } 0 \text {, } \\
\text { CH } 1\end{array}$ & $\begin{array}{l}\text { LS } 0 \text {, } \\
\text { CH } 0\end{array}$ & $\begin{array}{l}\text { LS } 0 \text {, } \\
\text { CH } 0\end{array}$ & $\begin{array}{l}\text { LS } 0 \text {, } \\
\text { CH } 0\end{array}$ & $\begin{array}{l}\text { LS } 0 \text {, } \\
\text { CH } 0\end{array}$ & LS $0, \mathrm{CH} 0$ & LS $0, \mathrm{CH} 0$ & LS $0, \mathrm{CH} 3$ \\
\hline 12 & $\begin{array}{l}\text { LS } 56, \\
\text { CH } 56\end{array}$ & $\begin{array}{l}\text { LS } 0 \text {, } \\
\text { CH } 0\end{array}$ & $\begin{array}{l}\text { LS } 0 \text {, } \\
\text { CH } 0\end{array}$ & $\begin{array}{l}\text { LS } 0 \text {, } \\
\text { CH } 5\end{array}$ & $\begin{array}{l}\text { LS 0, } \\
\text { CH } 0\end{array}$ & $\begin{array}{l}\text { LS 0, } \\
\text { CH } 0\end{array}$ & $\begin{array}{l}\text { LS 0, } \\
\text { CH } 0\end{array}$ & LS $0, \mathrm{CH} 0$ & LS $0, \mathrm{CH} 0$ & LS 0, CH 5 \\
\hline 22 & $\begin{array}{l}\text { LS 30, } \\
\text { CH } 27\end{array}$ & $\begin{array}{l}\text { LS } 0 \text {, } \\
\text { CH } 0\end{array}$ & $\begin{array}{l}\text { LS 1, } \\
\text { CH } 2\end{array}$ & $\begin{array}{l}\text { LS } 0 \text {, } \\
\text { CH } 0\end{array}$ & $\begin{array}{l}\text { LS } 0 \text {, } \\
\text { CH } 0\end{array}$ & $\begin{array}{l}\text { LS } 0 \text {, } \\
\text { CH } 0\end{array}$ & $\begin{array}{l}\text { LS 1, } \\
\text { CH } 0\end{array}$ & LS $0, \mathrm{CH} 1$ & LS $0, \mathrm{CH} 0$ & LS $3^{b}, \mathrm{CH} 3$ \\
\hline 13 & $\begin{array}{l}\text { LS 30, } \\
\text { CH } 31\end{array}$ & $\begin{array}{l}\text { LS 3, } \\
\text { CH } 3\end{array}$ & $\begin{array}{l}\text { LS 1, } \\
\text { CH } 2\end{array}$ & $\begin{array}{l}\text { LS 1, } \\
\text { CH } 2\end{array}$ & $\begin{array}{l}\text { LS } 0 \text {, } \\
\text { CH } 0\end{array}$ & $\begin{array}{l}\text { LS } 0, \\
\text { CH } 0\end{array}$ & $\begin{array}{l}\text { LS } 0, \\
\text { CH } 0\end{array}$ & LS $0, \mathrm{CH} 0$ & LS $0, \mathrm{CH} 0$ & LS 5, CH 7 \\
\hline 23 & $\begin{array}{l}\text { LS } 17, \\
\text { CH } 17\end{array}$ & $\begin{array}{l}\text { LS } 0, \\
\text { CH } 0\end{array}$ & $\begin{array}{l}\text { LS } 0 \text {, } \\
\text { CH } 0\end{array}$ & $\begin{array}{l}\text { LS } 0, \\
\text { CH } 0\end{array}$ & $\begin{array}{l}\text { LS } 0 \text {, } \\
\text { CH } 0\end{array}$ & $\begin{array}{l}\text { LS } 0 \text {, } \\
\text { CH } 0\end{array}$ & $\begin{array}{l}\text { LS } 0, \\
\text { CH } 0\end{array}$ & LS $0, \mathrm{CH} 0$ & LS $0, \mathrm{CH} 0$ & LS $0, \mathrm{CH} 0$ \\
\hline 16 & $\begin{array}{l}\text { LS } 42, \\
\text { CH } 42\end{array}$ & $\begin{array}{l}\text { LS; } 1 \text {, } \\
\text { CH } 1\end{array}$ & $\begin{array}{l}\text { LS 2, } \\
\text { CH } 5\end{array}$ & $\begin{array}{l}\text { LS 3, } \\
\text { CH } 5\end{array}$ & $\begin{array}{l}\text { LS } 0 \text {, } \\
\text { CH } 0\end{array}$ & $\begin{array}{l}\text { LS } 0 \text {, } \\
\text { CH } 0\end{array}$ & $\begin{array}{l}\text { LS 1, } \\
\text { CH } 2\end{array}$ & LS 2, CH 2 & LS $0, \mathrm{CH} 0$ & LS 9, CH 15 \\
\hline 24 & $\begin{array}{l}\text { LS 23, } \\
\text { CH } 22\end{array}$ & $\begin{array}{l}\text { LS } 0 \text {, } \\
\text { CH } 0\end{array}$ & $\begin{array}{l}\text { LS 1, } \\
\mathrm{CH} 2\end{array}$ & $\begin{array}{l}\text { LS } 0, \\
\mathrm{CH} 0\end{array}$ & $\begin{array}{l}\text { LS } 0 \text {, } \\
\text { CH } 0\end{array}$ & $\begin{array}{l}\text { LS } 0 \text {, } \\
\text { CH } 0\end{array}$ & $\begin{array}{l}\text { LS } 0 \text {, } \\
\mathrm{CH} 0\end{array}$ & LS $0, \mathrm{CH} 0$ & LS $0, \mathrm{CH} 0$ & LS $6, \mathrm{CH}^{\mathrm{c}}$ \\
\hline 8 & $\begin{array}{l}\text { LS } 125, \\
\text { CH } 125\end{array}$ & $\begin{array}{l}\text { LS 3, } \\
\text { CH } 7\end{array}$ & $\begin{array}{l}\text { LS } 1, \\
\text { CH } 2\end{array}$ & $\begin{array}{l}\text { LS } 0 \text {, } \\
\text { CH } 0\end{array}$ & $\begin{array}{l}\text { LS } 0 \text {, } \\
\text { CH } 0\end{array}$ & $\begin{array}{l}\text { LS 3, } \\
\text { CH } 3\end{array}$ & $\begin{array}{l}\text { LS } 1 \text {, } \\
\text { CH } 1\end{array}$ & LS $0, \mathrm{CH} 0$ & LS $0, \mathrm{CH} 0$ & LS 8, CH 13 \\
\hline 9 & $\begin{array}{l}\text { LS } 146, \\
\text { CH } 127\end{array}$ & $\begin{array}{l}\text { LS 6, } \\
\text { CH } 4\end{array}$ & $\begin{array}{l}\text { LS 1, } \\
\text { CH } 6\end{array}$ & $\begin{array}{l}\text { LS } 0, \\
\text { CH } 0\end{array}$ & $\begin{array}{l}\text { LS } 2, \\
\text { CH } 3\end{array}$ & $\begin{array}{l}\text { LS } 0, \\
\text { CH } 0\end{array}$ & $\begin{array}{l}\text { LS 2, } \\
\text { CH } 1\end{array}$ & LS 9, CH 8 & LS $0, \mathrm{CH} 0$ & $\mathrm{LS} 22, \mathrm{CH} 23^{\circ}$ \\
\hline
\end{tabular}

aPoor wound healing reported after 4 weeks of follow-up

${ }^{b}$ Chung et al. [22] reported fever (LS 1; CH 0)

cPattana-Arun et al.[24] reported wound dehiscence after 2 weeks of follow-up (LS 5, CH 4)

${ }^{\mathrm{d}}$ Altomare et al. [9] reported re-do surgery (LS 2; CH 1)

scores as assessed on a visual analogue scale on day 1 $[8,9,12,13,19,20,23,24]$, while the remaining studies reported pain scores without further time specification. The postoperative pain score was significantly reduced after LigaSure haemorrhoidectomy compared with conventional haemorrhoidectomy $(p=0.001$; Table 3 and Fig. 3) and it would take 491 studies to negate this significant result. Postoperative analgesia was quantitatively recorded in seven studies $[9,12$, $16,20-22,24]$, but the regime and method of assessment varied. Five of these studies reported significantly less need for postoperative analgesics after LigaSure haemorrhoidectomy [9, 12, 16, 20, 21]. Three studies reported no differences in postoperative analgesia requirements between the two groups [19, $22,24]$. 


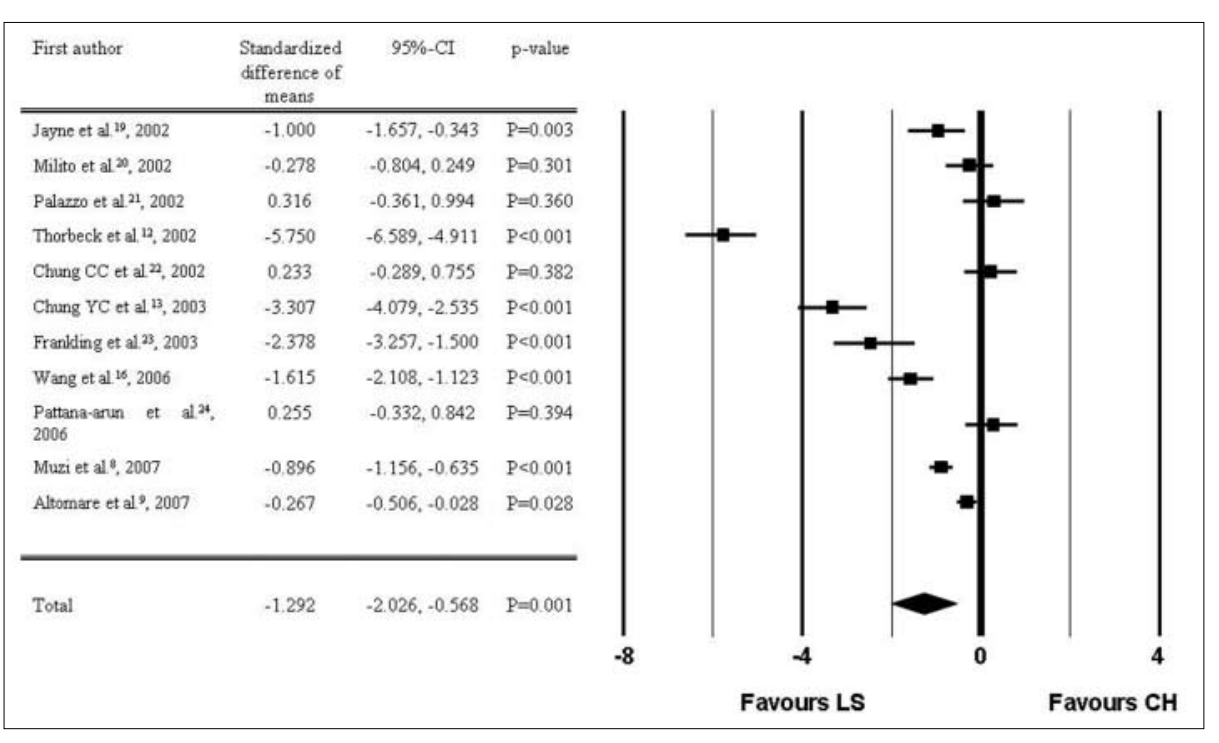

Fig. 3 Forest plot of postoperative pain score ( $L S$, LigaSure haemorrhoidectomy; $\mathrm{CH}$, conventional haemorrhoidectomy)

Fig. 4 Forest plot of time to return to normal activities ( $L S$, LigaSure haemorrhoidectomy; $\mathrm{CH}$, conventional haemorrhoidectomy)

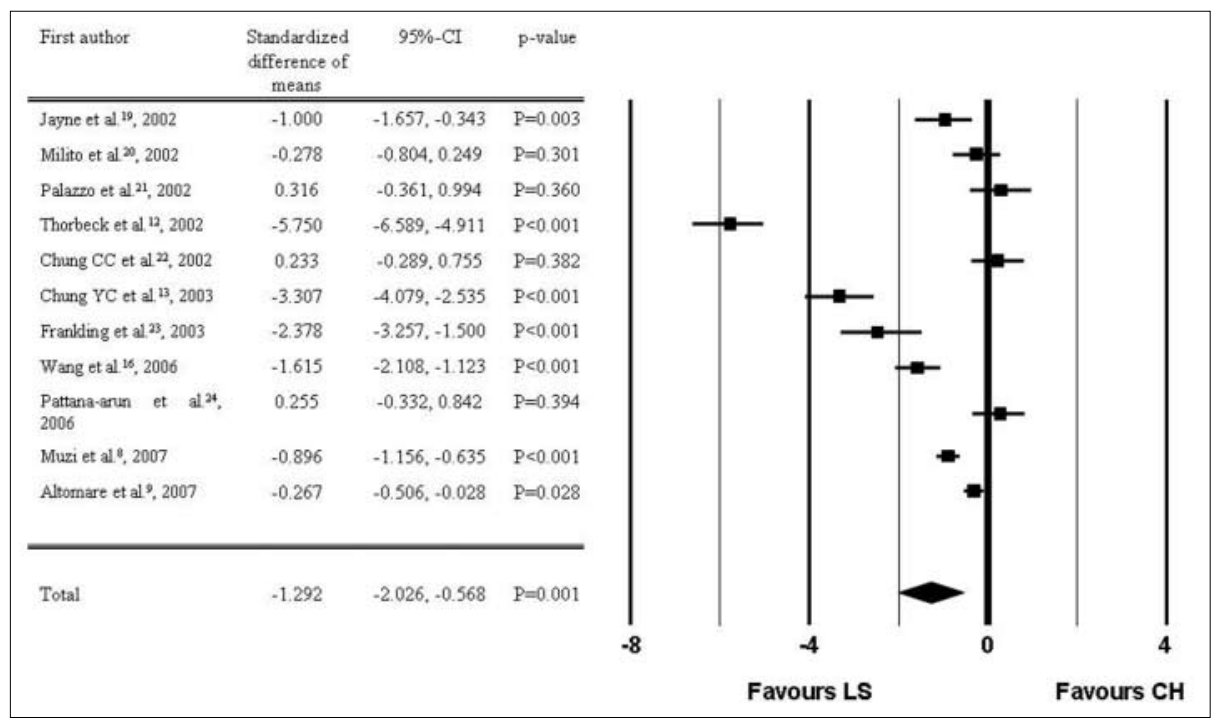

Wound healing and return to normal activities after LigaSure and conventional haemorrhoidectomy are also shown in Table 4. Wound healing was quantitatively reported by Milito et al. [20] and Muzi et al. [8]; whilst Thorbeck and Montes [12] noted that wound healing was "better and faster" after LigaSure haemorrhoidectomy. Chung and Wu [13] and Wang et al. [16] noted that wound healing was complete after 6 weeks in both groups. Quantitative meta-analysis confirmed that wound healing after LigaSure haemorrhoidectomy was significantly faster than after conventional haemorrhoidectomy ( $p=0.004$; Table 3). Milito et al. [20], Chung et al. [22], Wang et al. [16] Muzi et al. [8 ]and Altomare et al. [9] reported return to normal activities in a numerical format; while Jayne et al. [19] and Chung and $\mathrm{Wu}$ [13] reported no significant differences between the two groups without further quantification. Quantitative meta-analysis showed that after LigaSure haemorrhoidectomy, return to normal activities was significantly faster than after conventional haemorrhoidectomy ( $p=0.001$; Table 3 and Fig. 4 ) and it would take 185 studies to negate this result.

The follow-up in the clinical trials varied from 4 weeks $[9,24]$ to 36 months [8] (Table 4). Two studies reported longer follow-up periods in separate publications: Jayne et al. [19] at 36 months [17] and Palazzo et al. [21] at 15 months [18].

\section{Complications}

The overall incidence of complications reported (Table 5) was not significantly different after LigaSure haemorrhoidectomy than after conventional haemorrhoidectomy ( $p=0.056$; Table 3 and Fig. 5). At longer follow-up, patients who had had LigaSure haemorrhoidectomy were found to have significantly thicker internal anal sphincters as evaluated by endoanal ultrasonography than after conventional haemorrhoidectomy, 
Table 5 Results of quantitative meta-analysis of outcome characteristics based on 11 randomized controlled trials comparing LigaSure with conventional haemorrhoidectomy overall and stratified by open and closed conventional technique

\begin{tabular}{|c|c|c|c|c|c|}
\hline \multirow[t]{2}{*}{ Outcome } & \multirow{2}{*}{$\begin{array}{l}\text { Number of studies } \\
\text { (out of } 11)^{\mathrm{a}}\end{array}$} & \multirow{2}{*}{$\begin{array}{l}\text { Effect size } \\
{[95 \% \text { confidence interval]; }} \\
p \text {-value }\end{array}$} & \multirow{2}{*}{$\begin{array}{l}\text { Heterogeneity statistic } \\
(\mathrm{Q}) ; p \text {-value }\end{array}$} & \multicolumn{2}{|c|}{ Effect size [95\% confidence interval]; $p$-value } \\
\hline & & & & $\begin{array}{l}\text { Open } \\
\text { haemorrhoidectomy } \\
\text { (out of } 7 \text { ) }\end{array}$ & $\begin{array}{l}\text { Closed } \\
\text { haemorrhoidectomy } \\
\text { (out of } 3)^{\mathrm{b}}\end{array}$ \\
\hline \multirow[t]{2}{*}{$\begin{array}{l}\text { Duration of } \\
\text { operation (min) }\end{array}$} & 11 & $-2.2[-3.1,-1.4] ; 0.001$ & $321.7 ; 0.001$ & \multirow[t]{2}{*}{$\begin{array}{l}-2.1[-3.3,-0.9] \\
0.001\end{array}$} & \multirow[t]{2}{*}{$\begin{array}{l}-3.0[-4.8,-1.2] \\
0.001\end{array}$} \\
\hline & 9 & $-2.1[-3.0,-1.2] ; 0.001$ & $227.4 ; 0.001$ & & \\
\hline \multirow[t]{2}{*}{$\begin{array}{l}\text { In-hospital } \\
\text { stay (days) }\end{array}$} & $10^{c}$ & $-0.2[-0.6,0.1] ; 0.205$ & $57.6 ; 0.001$ & \multirow[t]{2}{*}{$\begin{array}{l}-0.1[-0.5,0.3] ; \\
0.608\end{array}$} & \multirow[t]{2}{*}{$\begin{array}{l}-0.5[-1.2,0.2] \\
0.136\end{array}$} \\
\hline & 8 & $-0.2[-0.7,0.2] ; 0.281$ & $54.0 ; 0.001$ & & \\
\hline \multirow[t]{2}{*}{$\begin{array}{l}\text { Postoperative } \\
\text { pain score }\end{array}$} & 11 & $-1.3[-2.0,-0.6] ; 0.001$ & $258.5 ; 0.001$ & \multirow[t]{2}{*}{$\begin{array}{l}-1.0[-1.9,-0.2] ; \\
0.018\end{array}$} & \multirow[t]{2}{*}{$\begin{array}{l}-2.4[-3.8,-1.1] \\
0.001\end{array}$} \\
\hline & 9 & $-0.6[-1.0,-0.1] ; 0.010$ & $71.8 ; 0.001$ & & \\
\hline \multirow{2}{*}{$\begin{array}{l}\text { Blood loss } \\
\text { during } \\
\text { operation }(\mathrm{ml})\end{array}$} & $4^{\mathrm{d}}$ & $-5.3[-8.3,-2.3] ; 0.001$ & $140.2 ; 0.001$ & \multirow[t]{2}{*}{$\begin{array}{l}-5.9[-10.8,-1.0] \\
0.019 ;(n=3)^{\mathrm{d}}\end{array}$} & \multirow[t]{2}{*}{$\begin{array}{l}-3.9[-12.4,4.5] \\
0.362(n=1)^{\mathrm{d}}\end{array}$} \\
\hline & $\begin{array}{l}3 \text { (excluding } \\
\text { Thorbeck and } \\
\text { Montes [12]) }\end{array}$ & $-4.8[-7.9,-1.6] ; 0.003$ & $76.2 ; 0.001$ & & \\
\hline $\begin{array}{l}\text { Wound healing } \\
\text { (days) }\end{array}$ & $2^{\mathrm{e}}$ & $-3.3[-5.5,-1.1] ; 0.004$ & $18.5 ; 0.001$ & $-^{\mathrm{e}}$ & - \\
\hline \multirow{3}{*}{$\begin{array}{l}\text { Return to } \\
\text { normal activities } \\
\text { Incidence of } \\
\text { complications }\end{array}$} & $5^{\mathrm{f}}$ & $-1.2[-2.0,-0.5] ; 0.001$ & $71.7 ; 0.001$ & \multirow{3}{*}{$\begin{array}{l}-0.9[-0.6,-0.3] ; \\
0.007(n=4)^{\mathrm{f}} \\
0.76[0.51,1.1] ; \\
0.166(n=7)\end{array}$} & \multirow{3}{*}{$\begin{array}{l}-2.4[-3.8,-1.0] \\
0.001(n=1)^{\mathrm{f}} \\
0.71[0.43,1.2] \\
0.185(n=3)^{\mathrm{g}}\end{array}$} \\
\hline & $10^{\mathrm{g}}$ & $0.74[0.55,1.0] ; 0.056$ & $5.3 ; 0.807$ & & \\
\hline & 8 & $0.76[0.55,1.1] ; 0.100$ & $3.2 ; 0.863$ & & \\
\hline
\end{tabular}

a First rows overall comparison for all studies which reported a specific outcome; second rows sensitivity analyses excluding the studies by Thorbeck and Montes [12] and Chung and $\mathrm{Wu}$ [13] because of concerns about the quality of the study design

${ }^{b}$ The study by Pattana-Arun et al. [24] was excluded from this analysis because the conventional haemorrhoidectomy used cannot be classified as either closed or open

'In-hospital stay was not reported by Pattana-Arun et al.[24]

dOnly Jayne et al. [19], Thorbeck and Wu [12], Chung et al. [22] and Wang et al. [16] recorded blood loss

e Only Milito et al. [20] and Muzi et al. [8] recorded wound healing numerically; both studies compared to open haemorrhoidectomy

fOnly Milito et al. [20], Chung et al. [22], Wang et al. [16], Muzi et al. [8] and Altomare et al. [9] recorded return to normal activities in a numerical format

gFrankling et al. [22] did not report any complications

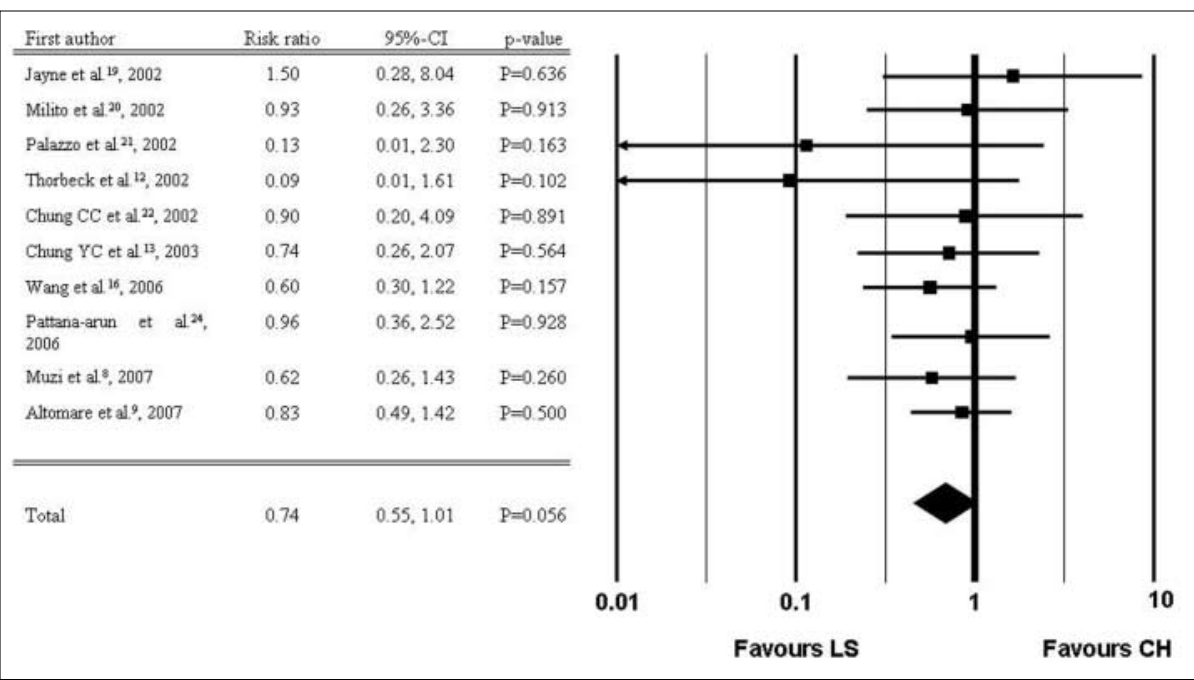

but there were no differences clinically at 36 months [17]. The other separate study with a 15-month followup period also reported no significant differences in clin- ical complications [18]. However, the numbers of patients who presented for reassessment in both studies were small [17, 18]. 
Sensitivity of meta-analysis, and stratification for open and closed conventional haemorrhoidectomy

Exclusion of the two studies $[12,13]$ that were considered to be of lesser design quality did not change the results of the meta-analysis. Throughout the quantitative meta-analysis numerical data heterogeneity remained significant. Differences in open and closed conventional haemorrhoidectomy did not affect results, apart from blood loss during operation which was no longer significant when LigaSure haemorrhoidectomy was compared to closed conventional haemorrhoidectomy ( $p=0.362$; Table 3). This result was based on the study of Wang et al. [16] only and would have been significant if fixed effect analysis had been assumed $(p<0.001)$.

\section{Discussion}

Evidence to date confirms that LigaSure haemorrhoidectomy is an effective technique. Our meta-analysis showed that LigaSure haemorrhoidectomy took significantly less time to complete. In addition, blood loss during LigaSure haemorrhoidectomy was significantly less. LigaSure appeared to be an effective tool for the dissection and haemostasis required for an excisional haemorrhoidectomy procedure. Some of the authors of the studies reviewed also remarked that the LigaSure method is comparatively simple and easy to learn [13, 19]. The postoperative pain score was significantly lower after LigaSure haemorrhoidectomy than after conventional haemorrhoidectomy. Most reports also confirmed a reduction in the amount of postoperative analgesia $[9,12,16,20,21]$, although this was documented in a non-uniform manner across the studies. Reduction in postoperative pain may be related to minimized tissue damage, improved tissue apposition promoting rapid primary healing [1] and temporary third degree burn injury to nerve endings at the site of the wound [25]. It is well accepted that postoperative pain is the aftermath most dreaded by patients undergoing haemorrhoidectomy [1]. However, LigaSure haemorrhoidectomy is more expensive than conventional haemorrhoidectomy because of the cost of the disposable basic Ligasure electrode which has a list price of US $\$ 304$. The estimated reduction in operation time of 2-3 min is not likely to result in significant cost savings. Our meta-analysis did not confirm any reduction in postoperative hospital stay. Hence, the added cost might only be justifiable for patients willing to pay extra for the estimated 1 to 2 points reduction on the 10point pain scale, and who are able to make good use of the estimated average 3 days earlier return to work.
Ligasure haemorrhoidectomy is a safe technique. Quantitative meta-analysis confirmed that wound healing after LigaSure haemorrhoidectomy was significantly faster. However, this did not lead to any significant reduction in the overall incidence of complications reported. The follow-up of the randomized controlled trials ranged from 4 weeks $[9,24]$ to 36 months [8]. Two studies reported longer follow-up periods in separate publications: Jayne et al. [19] at 36 months [17] and Palazzo et al. [21] at 15 months [18]. Complications at long-term follow-up including incontinence to flatus, continuing pain on defaecation, recurrent bleeding and patient dissatisfaction were reported. Unfortunately, the numbers available for analysis were not conclusive in favouring either LigaSure or conventional haemorrhoidectomy. The LigaSure system is claimed to precisely confine thermal spread to within $2 \mathrm{~mm}$ of the adjacent tissue [13]. Although this may lead to earlier wound healing, it does not seem to affect complication rates [27]. Theoretically, there is a worry that application of the Ligasure clamp may risk incorporating internal anal sphincter beneath the haemorrhoidal tissues. However, the very limited data to date have not shown any significant compromise of continence and the internal anal sphincter was found to be thicker on mid-term endoanal ultrasonography reassessment [17].

There has always been a matter of contention as to whether the advantages of a LigaSure haemorrhoidectomy are related mainly to a well-performed closed haemorrhoidectomy with primary closure and rapid healing of the wounds. However, stratified analysis has shown that the differences between open and closed conventional haemorrhoidectomy do not affect results, apart from blood loss during operation. Not surprisingly, the greater blood loss is associated mainly with the open technique. The haemorrhoidal pedicles were not ligated in most of the studies. Hence, there may be limited advantages in using a controlled diathermy sealing technique compared to suturing in primary closure after excisional haemorrhoidectomy.

Stapled haemorrhoidopexy is a conceptually different technique whereby the supplying blood vessels of the haemorrhoids are interrupted proximally [3]. In addition, the prolapsed anal cushions are excised proximally and reanchored by staples to an anatomically more correct position. Randomized controlled trials have confirmed advantages over both the open [3] and closed techniques of conventional excisional haemorrhoidectomy [26]. These advantages include less pain, shorter hospital stay and faster return to work or social activities [27]. Two randomized controlled studies have compared stapled haemorrhoidopexy with LigaSure haemorrhoidectomy. Kraemer et al. Have reported that stapled haemor- 
rhoidopexy results in less pain but higher risk of postoperative bleeding [28]. Basdanis et al. [29] have reported essentially no significant differences between stapled and LigaSure haemorrhoidectomy.

Since the previous meta-analysis published on this topic with 523 patients [7], two high-quality randomized controlled trials have accrued an additional 523 patients for analysis [8,9]. We can confirm the previous analysis findings that LigaSure haemorrhoidectomy leads to a shorter operative time, less blood loss and less pain at 24 hours. The recent additional patients have enabled clarification that after LigaSure haemorrhoidectomy return to normal work or social activity was significantly earlier, at a mean of approximately 3 days. Postoperative hospital stay and overall complications remained not significantly different between the two techniques. The present updated meta-analysis showed that LigaSure haemorrhoidectomy offers net benefits for the patient.

\section{References}

1. Ho Y-H, Buettner PG (2007) Open compared to closed hemorrhoidectomy: meta-analysis of randomized controlled trials. Tech Coloproctol 2:135-1343

2. Milligan ETC, Morgan CN, Jones LE, Officer R (1937) Surgical anatomy of the anal canal and the operative treatment of hemorrhoids. Lancet ii:1119-1124

3. Ho Y-H, Cheong W-K, Tsang C et al (2000) Stapled hemorrhoidectomy - cost and effectiveness. Randomized controlled trial including incontinence scoring, anorectal manometry, and endoanal assessments at up to three months. Dis Colon Rectum 43:1666-1675

4. Sayfan J, Becker A, Koltun L (2001) Sutureless closed hemorrhoidectomy: a new technique. Ann Surg 234:21-24

5. Armstrong DN, Ambroze WL, Schertzer ME, Orangio GR (2001) Harmonic Scalpel vs. electrocautery hemorrhoidectomy: a prospective evaluation. Dis Colon Rectum 44:558-564

6. Kennedy JS, Stranahan PL, Taylor KD, Chandler JG (1998) High-burst strength, feedback-controlled bipolar vessel sealing. Surg Endosc 12:876-878

7. Tan EK, Cornish J, Darzi AW et al (2007) Meta-analysis of shortterm outcomes of randomized controlled trials of LigaSure vs conventional hemorrhoidectomy. Arch Surg 142:1209-1218

8. Muzi MG, Milito G, Nigro C et al (2007) Randomized clinical trial of LigaSure and conventional diathermy hemorrhoidectomy. Br J Surg 94:937-942

9. Altomare DF, Milito G, Andreoli R et al (2008) Ligasure Precise vs. conventional diathermy for Milligan-Morgan hemorrhoidectomy: a prospective, randomized, multicenter trial. Dis Colon Rectum 51:514-519

10. Ferguson JA, Heaton JR (1959) Closed hemorrhoidectomy. Dis Colon Rectum 2:176-179

11. Mathai V, Ong BC, Ho YH (1996) Randomized controlled trial of lateral internal sphincterotomy with hemorrhoidectomy. Br J Surg 83:380-382

12. Thorbeck CV, Montes MF (2002) Hemorrhoidectomy: randomised controlled clinical trial of LigaSure compared with MilliganMorgan operation. Eur J Surg 168:482-484

13. Chung Y-C, Wu H-J (2003) Clinical experience of sutureless closed hemorrhoidectomy with LigaSure. Dis Colon Rectum 46:87-92

14. Moher D, Schulz KF, Alan DG (2001) The CONSORT statement: revised recommendations for improving the quality of reports of parallel-group randomised trials. Lancet 357:1191-1194

15. Lin LI (1989) A concordance correlation coefficient to evaluate reproducibility. Biometrics 45:255-268

16. Wang J-Y, Lu C-Y, Tsai H-L et al (2006) Randomized controlled trial of LigaSure with submucosal dissection versus Ferguson hemorrhoidectomy for prolapsed hemorrhoids. World J Surg 30:462-466

17. Peters CJ, Botterill I, Ambrose NS et al (2005) Ligasure trademark vs conventional diathermy hemorrhoidectomy: long-term followup of a randomised clinical trial. Colorectal Disease 7:350-353

18. Lawes DA, Palazzo FF, Francis DL, Clifton MA (2004) One year follow up of a randomized trial comparing Ligasure with open hemorrhoidectomy. Colorectal Dis 6:233-235

19. Jayne DG, Botterill I, Ambrose NS et al (2002) Randomized clinical trial of Ligasure versus conventional diathermy for day-case hemorrhoidectomy. Br J Surg 89:428-432

20. Milito G, Gargiani M, Cortese F (2002) Randomised trial comparing LigaSure hemorrhoidectomy with the diathermy dissection operation. Tech Coloproctol 6:171-175

21. Palazzo FF, Francis DL, Clifton MA (2002) Randomized clinical trial of Ligasure versus open hemorrhoidectomy. Br J Surg 89:154-157

22. Chung CC, Ha JP, Tai YP et al (2002) Double-blind randomized trial comparing harmonic scalpel hemorrhoidectomy, bipolar scissors hemorrhoidectomy, and scissors excision. Dis Colon Rectum 45:789-794

23. Franklin EJ, Seetharam S, Lowney J, Horgan PG (2003) Randomized, clinical trial of Ligasure vs conventional diathermy in hemorrhoidectomy. Dis Colon Rectum 46:1380-1383

24. Pattana-Arun J, Sooriprasoet N, Sahakijrungruang C et al (2006) Closed vs ligasure hemorrhoidectomy: a prospective, randomized clinical trial. J Med Assoc Thai 89:453-458

25. Seow-Choen F, Ho Y-H, Ang HG, Goh HS (1992) Prospective, randomised trial comparing pain and clinical function after conventional scissors excision/ligation vs diathermy excision without ligation of symptomatic prolapsed hemorrhoids. Dis Colon Rectum 35:1165-1169

26. Ho K-S, Ho Y-H (2006) Prospective randomized trial comparing stapled hemorrhoidopexy versus closed Ferguson hemorrhoidectomy. Tech Coloproctol 10:193-197

27. Shao WJ, Li GC, Zhang ZH et al (2008) Systematic review and meta-analysis of randomized controlled trials comparing stapled haemorrhoidopexy with conventional hemorrhoidectomy. Br J Surg 95:147-160

28. Kraemer M, Parulava T, Roblicj M et al (2005) Prospective, randomized study: Proximate ${ }^{\circledR}$ PPH stapler vs. Ligasure for hemorrhoidal surgery. Dis Colon Rectum 48:1517-1522

29. Basdanis G, Papadopoulos VN, Michalopoulos A et al (2005) Randomized clinical trial of stapled hemorrhoidectomy vs open with Ligasure for prolapsed piles. Surg Endosc 19:235-239 